\title{
Visual outcome and effect of treatment in stage III developing retrolental fibroplasia
}

\author{
C. G. KEITH \\ From the Royal Children's Hospital, Melbourne, Australia
}

SUMMARY Forty-five babies were seen with retrolental fibroplasia (RLF) stage III or more, that is, with a large vasoformative ridge protruding into the vitreous cavity. Thirty-four attended for follow-up, but subsequently 6 died. Of the remainder 12 had satisfactory vision, 3 unilateral and 8 bilateral high myopia, 3 were totally blind, and 2 were partially blind. It was found that stage III RLF could be subdivided according to its severity. Early stage III had a good prognosis, with 11 out of 18 achieving satisfactory vision, but only one out of 16 in RLF stage IIIa or more. Cryothermy was applied to 9 babies in the latter group, but it did not seem to influence the final outcome.

The acute or developing stages of retrolental fibroplasia (RLF) are followed by the stages of resolution and scarring, and it is well recognised that many cases of developing RLF resolve without causing any obvious ocular damage. However, if there is a large ridge of vasoformative tissue occupying one or more quadrants of the globe (stage III, Table 1), then the condition is likely to progress to some degree of permanent retinal damage. There have been several reports $^{1-7}$ on the use of laser coagulation, light coagulation, and cryopexy ${ }^{4}$ in order to try to arrest the progress of the disease, but the value of treatment is still not fully established. In this paper we discuss 45 cases of stage III RLF, 9 of which received treatment with cryothermy.

\section{Material and methods}

During a study ${ }^{8}$ on the incidence of RLF in 683 premature babies seen at the Royal Women's Hospital, Royal Children's Hospital, and Mercy Maternity Hospital, Melbourne, in the years 1977-9 developing RLF was found in 41 , and a further 2 were seen in the cicatricial stage. In 18 babies the condition did not reach beyond stage II, and in all these the retinal changes seemed to regress completely. However, the remaining 23 did reach stage III or more, and a significant number progressed to permanent retinal damage. These 23 plus the 2 babies seen in the cica-

Correspondence to Dr C. G. Keith, Royal Children's Hospital, Flemington Road, Parkville, Victoria 3052, Australia. tricial stage, and 20 more who were seen with stage III RLF after the end of the survey, are the subjects of this report.

The retinal changes in the developing stages were classified according to the scheme shown in Table 1 , while the cicatricial changes are graded according to the scheme in Table 2.9 At the outset of the survey it was considered that cryothermy should be given to any baby who reached stage IIIa, IIIb, or IV. Ten babies fell into this category, but one was too sick for

Table 1 RLF stages of development

Stage I Temporal vessels terminate in many parallel running finger-like branches

Abrupt margin between vascularised and nonvascularised retina temporally

Stage II Margin between vascularised and nonvascularised retina becomes slightly elevated to form a shelf or shunt in which the branching temporal vessels terminate

The peripheral vessels are dilated and often run parallel to the shelf

Stage III Shelf larger, projecting into vitreous, extending through one or two quadrants. Vascular channel visible in shelf. Moderate dilatation and tortuosity of peripheral vessels

Stage IIIa Sinusoids visible on surface of shunt. New blood vessels grow into vitreous. Increased generalised vascular engorgement. Shelf extends round $360^{\circ}$

Stage IIIb Vessels at posterior pole become very engorged and tortuous. Retina oedema. Haemorrhages. Marked vessel outgrowth into vitreous

Stage IV The shelf becomes larger protruding markedly into the vitreous. The dilatation of the vessels of the posterior pole may become extreme and they seem to arch into vitreous to reach the apex of the shelf. Localised retinal detachment may occur

Stage $V$ Total retinal detachment from disc to shelf or to ora serrata 
Table 2 RLF grades of resolution and scarring

Grade 0 Normal

Grade 1 Mild peripheral changes

Grade 2 Slight dragging of temporal vessels. Heterotopia of macula. Preretinal fibrosis. Myopia

Grade 3 Dragged disc with fibrous tissue in temporal periphery

Grade 4 Retinal fold with fibrous tissue on posterior surface of lens

Grade 5 Peripheral ring-shaped retinal detachment

Grade 6 Total funnel-shaped retinal detachment

Table 3 Visual status of 45 children who reached stage III developing RLF. Follow-up 8 months to 4 years

\begin{tabular}{lr}
\hline Visual status & No. \\
\hline Satisfactory & 15 \\
High myopia & 8 \\
Blind & 5 \\
Dead & 6 \\
No follow up & 11 \\
Total & 45 \\
\hline
\end{tabular}

treatment, so only 9 were actually treated, 6 bilaterally and 3 unilaterally. Cryothermy was not offered to any of the 20 babies born after 1979 , because of the doubtful benefit of the treatment. The procedure for cryothermy was as follows. The Keeler-Amoils cryothermy machine was set at $-70^{\circ} \mathrm{C}$, and multiple applications were made through the conjunctiva after a surface or local anaesthetic had been applied; general anaesthesia was not required. Treatment was directed to the vasoformative ridge itself, and not the avascular retina in front of it or the neovascularisation behind it. Most children received a single freeze-thaw cycle, but some received a double cycle. The probe was applied until the overlying retina was seen to be frozen.

The methods of examination in the premature nursery involved dilatation of the pupils with cyclopentolate $0.25 \%$ and phenylephrine $2.5 \%$ eyedrops, and the use of a premature infant speculum to separate the eyelids. The fundus was inspected with a binocular indirect ophthalmoscope and a $+30 \cdot 0$ DS lens, while the ora serrata was viewed with a custom-made scleral indenter. Follow-up examinations after the age of 6 months included checking the visual performance and assessing the ocular posture and mobility, refraction, and fundus examination after instilling cyclopentolate $1 \%$ drops. However, scleral indentation was not usually performed after one year of age.

\section{Results}

The visual outcome of 45 babies seen with stage III RLF is shown in Table 3. In 15 the visual status was classified as satisfactory, as the visual performance was normal for the age, and refraction showed no significant error. High myopia, more than $-9.0 \mathrm{DS}$, was found in 8 cases, and in several it increased rapidly over the first few years of observation. Five children were classified as blind, in 3 of whom the retinae were detached, and there was virtually no perception of light, while in 2 the visual acuity was less than $3 / 60$, and retinae were severely dragged. Eleven children were lost to follow-up, and 6 died.

Children in the treated group fared considerably worse than in the untreated or unselected groups. However, all of these were in RLF stage IIIa or more, and when they were compared with untreated patients of comparable severity (Table 4) there was little obvious difference between the 2 . Out of 9 children treated 8 survived, 2 are blind, 4 have high myopia, one unilateral high myopia, and only one is satisfactory, while of the 7 untreated children of comparable severity, 4 survived, 2 are blind, one has high myopia, and one has unilateral high myopia. Of the 18 children with early stage III RLF 16 survived, only one is blind, 3 have high myopia, one has unilateral high myopia, and 11 are satisfactory.

In Table 5 is shown a more detailed analysis of the ocular status in 33 children-the 28 who were followed up plus 5 of the children who died. It can be seen that only 14 eyes are normal (grade 0 ) out of a total of 66 . There are very minor peripheral changes in 11 eyes (grade 1), but even these may be predisposed to a retinal detachment in later life. Twentythree have a certain amount of vessel drag, and some have severe myopia (grade 2). Eyes in grades 3 to 4 have poor vision, while those in 5 or 6 have very little vision, and 18 eyes came into these 4 categories.

The gestational age and birth weight of the children

Table 4 Visual outcome of stage III RLF compared with that of treated and untreated stages IIIa, IIIb, and IV RLF

\begin{tabular}{|c|c|c|c|c|}
\hline & \multicolumn{2}{|l|}{ Untreated } & \multirow{2}{*}{$\frac{\text { Treated }}{\text { Stage III+ }}$} & \multirow[t]{2}{*}{ Total } \\
\hline & Stage III- & Stage III+ & & \\
\hline Satisfactory & 11 & 0 & 1 & 12 \\
\hline Myopia unilateral & 1 & 1 & 1 & 3 \\
\hline Myopia bilateral & 3 & 1 & 4 & 8 \\
\hline Socially blind & 0 & 1 & 1 & 2 \\
\hline Totally blind & 1 & 1 & 1 & 3 \\
\hline Dead & 2 & 3 & 1 & 6 \\
\hline Total & 18 & 7 & 9 & 34 \\
\hline
\end{tabular}

Table 5 Grades of cicatricial RLF found in the eyes of 33 babies who reached stage III RLF or more. Five of these babies subsequently died

\begin{tabular}{lrrrrrrr}
\hline Grade of RLF & 0 & $I$ & 2 & 3 & 4 & 5 & 6 \\
\hline Treated & - & 2 & 8 & 3 & 2 & 2 & 1 \\
Untreated & 4 & 5 & 9 & 2 & 2 & - & - \\
Unselected & 10 & 4 & 6 & 2 & - & - & 4 \\
Total & 14 & 11 & 23 & 7 & 4 & 2 & 5 \\
\hline
\end{tabular}




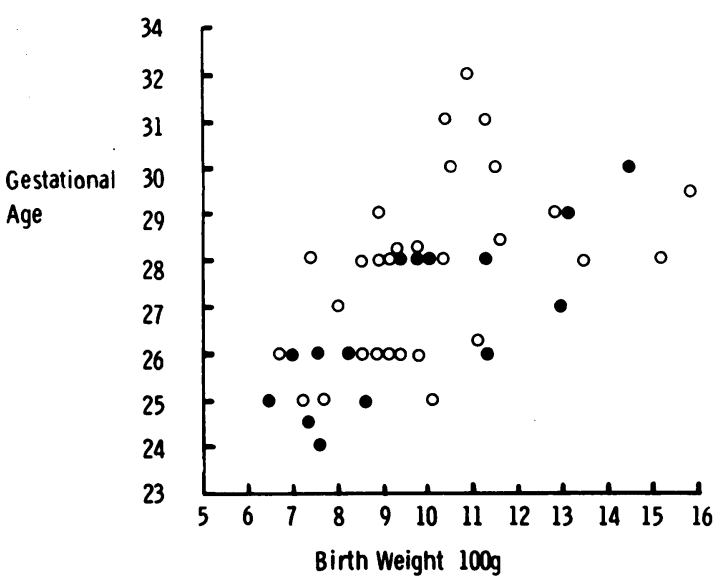

Fig. 1 Gestational age and birth weight of 45 babies who reached stage.III RLF. Those who eventually became blind or highly myopic are shown 0 .

are shown in Fig. 1. The fifteen who now have poor vision are separately marked, and it can be seen that 13 of them had a gestational age of less than 28 weeks, and most weighed less than $1000 \mathrm{~g}$ at birth. There was no particular tendency for small-for-dates babies to be more affected than others.

\section{Discussion}

The value of treatment in the acute stage of RLF is still not established because of the natural tendency for many cases to undergo resolution without a great deal of scarring, and it is not possible to determine in the early stages which ones will resolve and which will progress. Early reports on treatment were encouraging, ${ }^{1-4}$ but later ones have expressed doubt on its efficacy. ${ }^{56}$ The interpretation of many of the reports is difficult because of the lack of comparable classification schemes, and the established classification of Reese et al. ${ }^{10}$ does not correspond to the changes seen with the binocular indirect ophthalmoscope. Kingham $^{11}$ introduced a practical scheme based on indirect ophthalmoloscopy findings, and this has been modified ${ }^{9}$ to accord more with our own observations. Kingham" and Bensira $e t$ al. ${ }^{7}$ have used the term acute RLF as synonymous with developing RLF. However, in some of my cases the developing processes were quite slow to evolve, so I have not used this term. Basically Kingham' ${ }^{11}$ and Keith's' classification agree in that stages (grades) 1-11 developing RLF tend to resolve completely, while stage III is often followed by permanent damage, and stage IV and $\mathrm{V}$ are always followed by severe retinal damage. However, we have found that stage III needs to be subdivided into stage III, IIIa, and IIIb, depending on the extent of the circumferential shelf, the degree of vascular engorgement, and the amount of neovascularisation (Table 1 ). Out of 16 surviving stage III cases 11 have good vision, while only one case which reached stage IIIa or more is thought to have good vision (Table 4).

Treatment of the vasoformative ridge with cryothermy causes a rapid shrinkage of the shelf, with resolution of the proliferative changes in most cases, but significant scarring and retinal drag still occurred. However, it is not possible to say whether the regression would have been so marked without the cryothermy. On one case in stage IV no observable effect was obtained with the cryothermy. The cryothermy in one case that came to post-mortem had produced a satisfactory adhesion between retina and choroid. Laser and light coagulation have not been used in this series, as cryothermy seemed preferable, because of the hazy media and peripheral situation of the lesions. Kingham $^{5}$ reviewed the results of treating 12 infants with cryothermy; he concluded that it was probably deleterious or unnecessary and was apparently beneficial only in one case. Ben Sira ${ }^{7}$ reported on the good results of treatment, but in his cases the treatment was applied early, mainly in stage II or early stage III, so most of these would be expected to have undergone spontaneous resolution. He treated one eye initially and then treated the following eye if the condition continued to progress. In many of the cases the follow-up was short (20 weeks), and we have found in many of our cases that initially the results were good, but later high myopia developed. Further problems in deciding when to treat are highlighted by 2 babies who did not follow the usual course, as the shelf was only slightly elevated above the level of the retina, with very little general vascular engorgement, but the peripheral changes went on to severe fibrosis and total retinal detachment in one child, while in another it resulted in grade II scarring.

The first 23 cases of stage III RLF (plus 2 cases seen in the cicatricial stage only) were derived from the screening of 358 babies weighing less than $1500 \mathrm{~g}$, giving a prevalence of $7 \cdot 0 \%$. The overall risk of blindness was small, as this was found in only 3 babies $(0 \cdot 8 \%)$. Among the 358 babies seen 86 weighed less than $1000 \mathrm{~g} ; 12$ of these reached stage III RLF, one $(1.2 \%)$ became blind, and $5(6 \%)$ highly myopic.

The danger of visual impairment in surviving premature babies is not very high, but it increases with increased prematurity (Fig. 1) and is quite significant if stage III developing RLF is reached, as out of 28 survivors $5(18 \%)$ were blind. It would seem that, while RLF is no longer a very common cause of blindness within the community, it is still a personal tragedy for the affected child and his parents. 
I am indebted to Mrs S. Smith for clinical assistance and Ms Gayle Moore for secretarial help.

\section{References}

1 Payne J, Patz A. Treatment of acute proliferative retrolental fibroplasia. Trans Am Acad Ophthalmol Otolaryngol 1972; 76: 1234-46.

2 O'Grady GE, Flynn JT, Herrara JA. The clinical course of retrolental fibroplasia in premature infants. South Med J 1972; 65: 655-8.

3 Nagata M. Tsuruoka Y. Treatment of acute retrolental fibroplasia with xenon arc photogoaculation. Jpn J Ophthalmol 1972; 16: 131-43.

4 Oshima K, Ikui H, Kano M, et al. Clinical study and photocoagulation of retinopathy of prematurity. Folia Ophthalmol Jpn 1971; 22: 7000 .
5 Kingham JD. Acute retrolental fibroplasia. II. treatment by cryosurgery. Arch Ophthalmol 1978; 96: 2049-53.

6 Kalina RE. Treatment of retrolental fibroplasia. Surv Ophthalmol 1980; $24: 229-36$.

7 Ben-Sira I, Nissenkorn I, Grunwald E, Yassur Y. Treatment of acute retrolental fibroplasia by cryopexy. Br J Ophthalmol 1980; 64: 758-62.

8 Keith CG, Smith S, Lansdell B. Retrolental fibroplasia: a study of the incidence and aetiological factors 1977-1979. Med J Aust 1981 ; ii: 589-92.

9 Keith CG. Retrolental fibroplasia, a new classification of the developing and cicatricial changes. Aust J Ophthalmol 1979; 7: 189-94.

10 Reese AB, King M, Owens WC. Classification of retrolental fibroplasia. Am J Ophthalmol 1953; 36: 1333-5.

11 Kingham JD. Acute retrolental fibroplasia. Arch Ophthalmol 1977; 95: 39-47. 